\title{
Logics for Reversible Regular Languages and Semigroups with Involution
}

Paul Gastin

LSV, ENS Paris-Saclay \& CNRS, Université Paris-Saclay, France

paul.gastin@lsv.fr

\author{
Amaldev Manuel \\ Indian Institute of Technology Goa, India \\ amal@iitgoa.ac.in
}

\section{R. Govind}

Chennai Mathematical Institute, Chennai, India and LaBRI, University of Bordeaux, France govindr@cmi.ac.in

\begin{abstract}
We present MSO and FO logics with predicates 'between' and 'neighbour' that characterise various fragments of the class of regular languages that are closed under the reverse operation. The standard connections that exist between MSO and FO logics and varieties of finite semigroups extend to this setting with semigroups extended with an involution. The case is different for FO with neighbour relation where we show that one needs additional equations to characterise the class.
\end{abstract}

2012 ACM Subject Classification Theory of computation $\rightarrow$ Formal languages and automata theory; Theory of computation $\rightarrow$ Logic

Keywords and phrases Regular languages, reversible languages, first-order logic, automata, semigroups

Funding Partly supported by UMI ReLaX.

\section{Introduction}

In this paper we look closely at the class of regular languages that are closed under the reverse operation. We fix a finite alphabet $A$ for the rest of our discussion. The set $A^{*}$ (respectively $A^{+}$) denotes the set of all (resp. non-empty) finite words over the alphabet $A$. If $w=a_{1} \cdots a_{k}$ with $a_{i} \in A$ is a word then $w^{r}=a_{k} \cdots a_{1}$ denotes the reverse of $w$. This notion is extended to sets of words pointwise, i.e. $L^{r}=\left\{w^{r} \mid w \in L\right\}$ and we can talk about reverse of languages. A regular language $L \subseteq A^{*}$ is closed under reverse or simply reversible if $L^{r}=L$. We let Rev denote the class of all reversible regular languages. Clearly Rev is a strict subset of the class of all regular languages.

The class Rev is easily verified to be closed under union, intersection and complementation. It is also closed under homomorphic images, and inverse homomorphic images under alphabetic (i.e. length preserving) morphisms. However they are not closed under quotients. For instance, the language $L=(a b c)^{*}+(c b a)^{*}$ is closed under reverse but the quotient $a^{-1} L=b c(a b c)^{*}$ is not closed under reverse. Thus the class Rev fails to be a variety of languages - i.e. a class closed under Boolean operations, inverse morphic images and quotients. However reversible languages are closed under bidirectional quotients, i.e. quotients of the form $u^{-1} L v^{-1} \cup\left(v^{r}\right)^{-1} L\left(u^{r}\right)^{-1}$, given words $u, v$. Thus, to a good extent, Rev shares properties similar to that of regular languages. Hence it makes sense to ask the question

"are there good logical characterisations for the class Rev and its well behaved subclasses?". 
Our results. We suggest a positive answer to the above question. We introduce two predicates between (bet $(x, y, z)$ is true if position $y$ is between positions $x$ and $z$ ) and neighbour ( $\mathrm{N}(x, y)$ is true if positions $x$ and $y$ are adjacent). The predicates between and neighbour are the natural analogues of the order relation $<$ and successor relation +1 in the undirected case. In fact this analogy extends to the case of logical definability. We show that Rev is the class of monadic second order (MSO) definable languages using either of the predicates, i.e. MSO(bet) or $\mathrm{MSO}(\mathrm{N})$. This is analogous to the classical Büchi-ElgotTrakhtenbrot theorem relating regular languages and MSO logic. This connection extends to the case of first order logic as well. We show that $\mathrm{FO}$ (bet) definable languages are precisely the reversible languages definable in $\mathrm{FO}(<)$. However the case of successor relation is different, i.e. the class of $\mathrm{FO}(\mathrm{N})$ definable languages is a strict subset of reversible languages definable in $\mathrm{FO}(+1)$. The precise characterisation of this class is one of our main contributions.

The immediate question that arises from the above characterisations is one of definability: Given a reversible language is it definable in the logic?". The case of $\mathrm{FO}$ (bet) is decidable due to Schützenberger-McNaughton-Papert theorem that states that syntactic monoids of $\mathrm{FO}(<)$ definable languages are aperiodic (equivalent to the condition that the monoid contains no groups as subsemigroups) [9, 8. However the question for $\mathrm{FO}(\mathrm{N})$ is open. We prove a partial characterisation in terms of semigroups with involution. It is to be noted that the characterisation of $\mathrm{FO}(+1)$ is a tedious one that goes via categories [11].

Related work. A different but related between predicate (namely $a(x, y)$, for $a \in A$, is true if there is an $a$-labelled position between positions $x$ and $y$ ) was introduced and studied in [5, 6, 7. Such a predicate is not definable in $\mathrm{FO}^{2}(<)$, the two variable fragment of first-order logic (which corresponds to the well known semigroup variety DA [12]). The authors of [5, 6, 7] study the expressive power of $\mathrm{FO}^{2}(<)$ enriched with the between predicates $a(x, y)$ for $a \in A$, and show an algebraic characterisation of the resulting family of languages. The between predicate (predicates rather) in [5] is strictly less expressive than the between predicate introduced in this paper. However the logics considered in [5] have the between predicates in conjunction with order predicates $<$ and +1 . Hence their results are orthogonal to ours.

Another line of work that has close parallels with the one in this paper is the variety theory of involution semigroups (also called $\star$-semigroups) (see [3] for a survey). Most investigations along these lines have been on subvarieties of regular $\star$-semigroups (i.e. $\star$-semigroups satisfying the equation $x x^{\star} x=x$ ). As far as we are aware the equation introduced in this paper has not been studied before.

Structure of the paper. In Section 2 we introduce the predicates and present our logical characterisations. This is followed by a characterisation of $\mathrm{FO}(\mathrm{N})$. In Section 3 we discuss semigroups with involution, a natural notion of syntactic semigroups for reversible languages. In Section 4 we conclude.

\section{Logics with Between and Neighbour}

As usual we represent a word $w=a_{1} \cdots a_{n}$ as a structure containing positions $\{1, \ldots, n\}$, and unary predicates $P_{a}$ for each letter $a$ in the alphabet. The predicate $P_{a}$ is precisely true at those positions labelled by letter $a$. The atomic predicate $x<y($ resp. $x+1=y)$ is true if position $y$ is after (resp. immediately after) position $x$. The logic FO is the logic containing atomic predicates, boolean combinations $(\phi \vee \psi, \phi \wedge \psi, \neg \psi$ whenever $\phi, \psi$ are formulas of the 
logic), and first order quantifications ( $\exists x \psi, \forall x \psi$ if $\psi$ is a formula of the logic). The logic MSO in addition contains second order quantification as well $(\exists X \psi, \forall X \psi$ if $\psi$ is a formula of the logic) - i.e. quantification over sets of positions. By $\operatorname{FO}(\tau)$ or $\operatorname{MSO}(\tau)$ we mean the corresponding logic with atomic predicates $\tau$ in addition to the unary predicates $P_{a}$. The classical result relating $\mathrm{MSO}$ and regular languages states that $\mathrm{MSO}(<)=\mathrm{MSO}(+1)$ defines all regular languages. We introduce two analogous predicates for the class Rev of reversible regular languages.

\section{1 $\mathrm{MSO}$ (bet), $\mathrm{MSO}(\mathrm{N})$ and $\mathrm{FO}($ bet $)$}

The ternary between predicate bet $(x, y, z)$ is true for positions $x, y, z$ when $y$ is in between $x$ and $z$, i.e.

$$
\operatorname{bet}(x, y, z):=x<y<z \text { or } z<y<x
$$

- Example 1. The set of all words containing the subword $a_{1} a_{2} \cdots a_{k}$ or $a_{k} a_{k-1} \cdots a_{1}$ is defined by the formula

$$
\exists x_{1} \exists x_{2} \cdots \exists x_{k} \bigwedge_{i=1}^{k} P_{a_{i}}\left(x_{i}\right) \wedge \bigwedge_{i=2}^{k-1} \operatorname{bet}\left(x_{i-1}, x_{i}, x_{i+1}\right)
$$

The 'successor' relation of bet is the binary predicate neighbour $\mathrm{N}(x, y)$ that holds true when $x$ and $y$ are neighbours, i.e.

$$
\mathrm{N}(x, y):=x+1=y \text { or } y+1=x
$$

- Example 2. The set of words of even length is defined by the formula

$$
\exists X\left(X\left(e_{1}\right) \wedge \neg X\left(e_{2}\right) \wedge \forall x \forall y(\mathrm{~N}(x, y) \rightarrow(X(x) \leftrightarrow \neg X(y)))\right)
$$

where $e_{1}, e_{2}$ are the endpoints, i.e. the two positions with exactly one neighbour (defined easily in $\mathrm{FO}(\mathrm{N}))$.

The relation $\mathrm{N}(x, y)$ can be defined in terms of bet using first-order quantifiers as $x \neq$ $y \wedge \forall z \neg$ bet $(x, z, y)$. One can also define bet $(x, y, z)$ in terms of $\mathrm{N}$, but using second-order set quantification. To do this we assert that any subset $X$ of positions

- that contains $x, z$ and at least some other position

- and such that any position in $X$, except for $x$ and $z$, has exactly two neighbours in $X$, contains the position $y$.

- Proposition 3. For definable languages, $\mathrm{MSO}($ bet $)=\mathrm{MSO}(\mathrm{N})=\operatorname{Rev}$.

Proof. Clearly from the discussion above, $\mathrm{MSO}($ bet $)=\mathrm{MSO}(\mathrm{N}) \subseteq$ Rev. To show the other inclusion, let $L$ be a reversible regular language and let $\varphi$ be a formula in $\mathrm{MSO}(<)$ defining it. Pick an endpoint $e$ of the given word, an endpoint is a position with exactly one neighbour, a property expressible in $\mathrm{FO}(\mathrm{N}) \subseteq \mathrm{FO}$ (bet). We relativize the formula $\varphi$ with respect to $e$ by replacing all occurrences of $x<y$ in the formula by $(e=x \neq y) \vee \operatorname{bet}(e, x, y)$. Let $\varphi^{\prime}(e)$ be the formula obtained in this way and let $\psi(e)=\neg \exists x, y(x \neq y \wedge \mathrm{N}(e, x) \wedge \mathrm{N}(e, y))$ be the $\mathrm{FO}(\mathrm{N})$ formula asserting that $e$ is an endpoint, then we claim that

$$
\chi=\exists e\left(\psi(e) \wedge \varphi^{\prime}(e)\right)
$$


defines the language $L$. Let $w$ be a word of length $k \geq 1$ then,

$$
\begin{aligned}
w \models \chi & \Leftrightarrow w, 1 \models \varphi^{\prime}(e) \text { or } w, k \models \varphi^{\prime}(e) \\
& \Leftrightarrow w \models \varphi \text { or } w^{r} \models \varphi \\
& \Leftrightarrow w \models \varphi \quad \text { (since } L \text { is reversible) }
\end{aligned}
$$

Hence $L(\chi)=L(\varphi)=L$.

The above proposition says that $\mathrm{MSO}($ bet $)=\mathrm{MSO}(<) \cap \operatorname{Rev}$. This carries down to the first-order case using the same relativization idea. In fact the result holds for the prefix class $\Sigma_{i}$ (first-order formulas in prenex normal form with $i$ blocks of alternating quantifiers starting with $\exists$-block).

- Proposition 4. The following is true for definable languages.

1. $\mathrm{FO}($ bet $)=\mathrm{FO}(<) \cap \operatorname{Rev}$.

2. $\Sigma_{i}$ (bet $)=\Sigma_{i}(<) \cap \operatorname{Rev}$.

Proof. Given an $\mathrm{FO}(<)$ formula in prenex form defining a language in Rev, we replace every occurrence of $x<y$ by $(e=x \neq y) \vee$ bet $(e, x, y)$ as before, where $e$ is asserted to be an endpoint with $\psi(e)=\forall x, y \neg$ bet $(x, e, y)$. For every formula in $\Sigma_{i}(<), i \geq 2$ this results in an equivalent formula in $\Sigma_{i}$ (bet). For the case of $\Sigma_{1}$, let us note that every formula in $\Sigma_{1}(<)$ defines a union of languages of the form $A^{*} a_{1} A^{*} a_{2} A^{*} \cdots A^{*} a_{k} A^{*}$. Such a language can be written as a disjunction of formulas like the one in Example 1 .

\section{$2.2 \mathrm{FO}(\mathrm{N})$}

Next we address the expressive power of FO with the neighbour predicate.

We start by detailing the class of locally threshold testable languages. Recall that word $y$ is a factor of word $u$ if $u=x y z$ for some $x, z$ in $A^{*}$. We use $\sharp(u, y)$ to denote the number of times the factor $y$ appears in $u$.

Let $\approx_{k}^{t}$, for $k, t>0$, be the equivalence on $A^{*}$, whereby two words $u$ and $v$ are equivalent if either they both have length at most $k-1$ and $u=v$, or otherwise they have

1. the same prefix of length $k-1$,

2. the same suffix of length $k-1$,

3. and the same number of occurrences, upto threshold $t$, for all factors of length $\leq k$, i.e. for each word $y \in A^{*}$ of length at most $k$, either $\sharp(u, y)=\sharp(v, y)<t$, or $\sharp(u, y) \geq t$ and $\sharp(v, y) \geq t$.

- Example 5. We have $a b a b a b \approx_{2}^{1} a b a b \neq_{2}^{1} a b b a b$. Indeed, all the words start and end with the same letter. In the first two words the factors $a b$ as well as $b a$ appear at least once. While in the last word the factor $b b$ appears once while it is not present in the word $a b a b$. Notice also that $a b a b a b \neq_{2}^{2} a b a b$ due to the factor $b a$.

A language is locally threshold testable (or LTT for short) if it is a union of $\approx_{k}^{t}$ classes, for some $k, t>0$.

- Example 6. The language $(a b)^{*}$ is LTT. In fact it is locally testable (the special case of locally threshold testable with $t=1)$. Indeed, $(a b)^{*}$ is the union of three classes: $\{\varepsilon\},\{a b\}$ and $a b a b(a b)^{*}$ which is precisely the set of words that begin with $a$, end with $b$, and the only factors are $a b$ and $b a$.

A language that is definable in $\mathrm{FO}(<)$ and not LTT is $c^{*} a c^{*} b c^{*}$. In this language if $a$ and $b$ are sufficiently separated by $c$-blocks then the order between $a$ and $b$ cannot be 
differentiated. It can be proved that for any $t, k$ there is a sufficiently large $n$ such that $c^{n} a c^{n} b c^{n} \approx_{k}^{t} c^{n} b c^{n} a c^{n}$.

Locally threshold testable languages are precisely the class of languages definable in $\mathrm{FO}(+1)$ [1, 13]. Since we can define the neighbour predicate $\mathrm{N}$ using +1 , clearly $\mathrm{FO}(\mathrm{N}) \subseteq$ $\mathrm{FO}(+1) \cap \operatorname{Rev}=\mathrm{LTT} \cap$ Rev. But this inclusion is strict as shown in Example 8.

- Example 7. Consider the language $L=u a^{*}+a^{*} u^{r}$ of words which have either $u$ as prefix

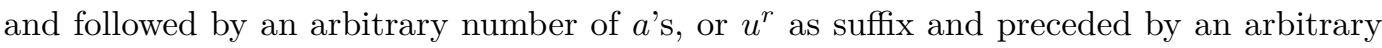
number of $a$ 's. The language $L$ is in $\mathrm{FO}(\mathrm{N})$. When $u=a_{1} \cdots a_{n}$, it can be defined by a formula of the form $\exists x_{1}, \ldots, x_{n} \psi$ where $\psi$ states that $x_{1}$ is an endpoint, $\bigwedge_{1 \leq i<n} \mathrm{~N}\left(x_{i}, x_{i+1}\right)$, $\bigwedge_{1<i<n} x_{i-1} \neq x_{i+1}, \bigwedge_{1 \leq i \leq n} P_{a_{i}}\left(x_{i}\right)$, and all other positions are labelled $a$.

- Example 8. Consider the language $L$ over the alphabet $\{a, b, c\}$,

$$
L=\{w \mid \sharp(w, a b)=2, \sharp(w, b a)=1 \text { or } \sharp(w, a b)=1, \sharp(w, b a)=2\} \text {. }
$$

Since $L$ is locally threshold testable and reverse closed, $L \in \mathrm{FO}(+1) \cap \operatorname{Rev}$.

We can show that $L \notin \mathrm{FO}(\mathrm{N})$ by showing that the words,

$$
c^{k} a b c^{k} b a c^{k} a b c^{k} \in L \quad \quad c^{k} a b c^{k} a b c^{k} a b c^{k} \notin L
$$

for $k>0$ are indistinguishable by an $\mathrm{FO}(\mathrm{N})$ formula of quantifier depth $k$. For showing the latter claim, one uses Ehrenfeucht-Fraissé games and argues that in the $k$-round EF-game the duplicator has a winning strategy. The strategy is roughly described below:

$$
\underline{c^{k} a b c^{k} b} a c^{k} a b c^{k} \quad c^{k} a b c^{k} a \underline{b c^{k} a b c^{k}}
$$

Any move of the spoiler is mimicked by the duplicator in the corresponding underlined or non-underlined part of the other word, while maintaining the neighbourhood relation between positions. For instance, if the spoiler plays the first $b$ on the underlined part of the first word, then the duplicator chooses the last $b$ on the underlined portion of the word on the right. Similarly, if the spoiler plays the first $a$ on the non-underlined part of the first word, the duplicator chooses the last $a$ on the non-underlined portion of the word on the right. Note that, since no order on positions in the words can be checked with the neighbour predicate, there is no way to distinguish between these words, if the duplicator plays in the above way ensuring that the position played has the same neighbourhood relation as the position played by the spoiler. Therefore, the Neighbour predicate will not be able to distinguish between $a b$ and $b a$ when they are sufficiently separated by $c$ 's.

From the above example, we get,

- Proposition 9. For definable languages, $\mathrm{FO}(\mathrm{N}) \subsetneq \mathrm{FO}(+1) \cap \operatorname{Rev}=\mathrm{LTT} \cap \operatorname{Rev}$.

Next we will characterise the class of languages accepted by $\mathrm{FO}(\mathrm{N})$. For $t>0$ we define the equality with threshold $t$ on the set $\mathbb{N}$ of natural numbers by $i={ }^{t} j$ if $i=j$ or $i, j \geq t$. Recall that $\sharp(w, v)$ denotes the number of occurrences of $v$ in $w$, i.e. the number of pairs $(x, y)$ such that $w=x v y$. We extend this to $\sharp^{r}(w, v)$ which counts the number of occurrences of $v$ or $v^{r}$ in $w$, i.e. the number of pairs $(x, y)$ such that $w=x v y$ or $w=x v^{r} y$. Notice that $\sharp^{r}(w, v)=\sharp^{r}\left(w, v^{r}\right)=\sharp^{r}\left(w^{r}, v\right)=\sharp^{r}\left(w^{r}, v^{r}\right)$.

We define now the locally-reversible threshold testable (LRTT) equivalence relation. Let $k, t>0$. Two words $w, w^{\prime} \in A^{*}$ are $(k, t)$-LRTT equivalent, denoted $w^{r} \stackrel{r}{\approx}_{k}^{t} w^{\prime}$ if $|w|<k$ and $w^{\prime} \in\left\{w, w^{r}\right\}$, or 
- $w, w^{\prime}$ are both of length at least $k$, and

- $\sharp^{r}(w, v)=^{t} \sharp^{r}\left(w^{\prime}, v\right)$ for all $v \in A^{\leq k}$, and

- if $x, x^{\prime}$ are the prefixes of $w, w^{\prime}$ of length $k-1$ and $y, y^{\prime}$ are the suffixes of $w, w^{\prime}$ of length $k-1$ then $\left\{x, y^{r}\right\}=\left\{x^{\prime}, y^{\prime r}\right\}$.

Notice that $w \stackrel{r}{\approx}{ }_{k}^{t} w^{r}$ for all $w \in A^{*}$ and $w \approx_{k}^{t} w^{\prime}$ implies $w \stackrel{r}{\approx}_{k}^{t} w^{\prime}$ for all $w, w^{\prime} \in A^{*}$. Notice

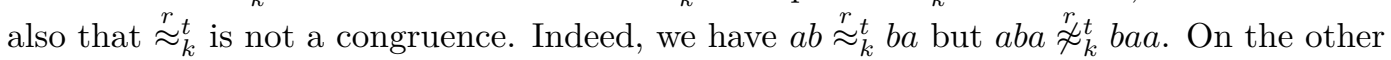
hand, if $v \stackrel{r}{\approx} t k w$ then for all $u \in A^{*}$ we have $u v \stackrel{r}{\approx}_{k}^{t} u w$ or $u v \stackrel{r}{\approx}_{k}^{t} u w^{r}$, and similarly $v u \stackrel{r}{\approx}_{k}^{t} w u$ or $v u \stackrel{r}{*} w_{k}^{r} u$.

- Definition 10 (Locally-Reversible Threshold Testable Languages). A language L is locallyreversible threshold testable, LRTT for short, if it is a union of equivalence classes of $\stackrel{r}{*}_{k}^{t}$ for some $k, t>0$.

- Theorem 11. Languages defined by $\mathrm{FO}(\mathrm{N})$ are precisely the class of locally-reversible threshold testable languages.

Proof. $(\Leftarrow)$ Assume we are given an LRTT language, i.e. a union of $\stackrel{r}{\approx}_{k}^{t}$-classes for some $k, t>0$. We explain how to write an $\mathrm{FO}(\mathrm{N})$ formula for each $\stackrel{r}{\approx}{ }_{k}^{t}$-class. Consider a word $v=a_{1} a_{2} \cdots a_{n} \in A^{+}$. For $m \in \mathbb{N}$, we can say that $v$ or its reverse occurs at least $m$ times in a word $w \in A^{*}$, i.e. $\sharp^{r}(w, v) \geq m$, by the formula

$$
\begin{aligned}
\varphi_{v}^{\geq m}= & \exists x_{1,1} \cdots \exists x_{1, n} \cdots \exists x_{m, 1} \cdots \exists x_{m, n} \\
& \bigwedge_{i=1}^{m}\left(\bigwedge_{j=1}^{n-1} \mathrm{~N}\left(x_{i, j}, x_{i, j+1}\right) \wedge \bigwedge_{j=2}^{n-1}\left(x_{i, j-1} \neq x_{i, j+1}\right) \wedge \bigwedge_{j=1}^{n} P_{a_{j}}\left(x_{i, j}\right)\right) \\
& \wedge \bigwedge_{1 \leq i<j \leq m} \neg\left(\left(x_{i, 1}=x_{j, 1} \wedge x_{i, n}=x_{j, n}\right) \vee\left(x_{i, 1}=x_{j, n} \wedge x_{i, n}=x_{j, 1}\right)\right) .
\end{aligned}
$$

Similarly, we can write a formula $\psi_{v} \in \mathrm{FO}(\mathrm{N})$ that says that a word belongs to $\left\{v, v^{r}\right\}$. Finally, given two words of same length $u, v \in A^{n}$, we can write a formula $\chi_{u, v} \in \mathrm{FO}(\mathrm{N})$ that says that $u, v$ occur at two different end points of a word $w$, i.e. that $\left\{x, y^{r}\right\}=\{u, v\}$ where $x, y$ are the prefix and suffix of $w$ of length $n$.

$(\Rightarrow)$ Hanf's theorem [4] states that two structures $A$ and $B$ are $m$-equivalent (i.e. indistinguishable by any FO formula of quantifier rank at most $m$ ), for some $m \in \mathbb{N}$ if for each $3^{m}$ ball type $S$, both $A$ and $B$ have the same number of $3^{m}$ balls of type $S$ upto a threshold $m \times e$, where $e \in \mathbb{N}$. Applying Hanf's theorem to undirected path graphs, we obtain that given an $\mathrm{FO}(\mathrm{N})$ formula $\Phi$, there exist $k, t>0$ such that the fact that a word $w$ satisfies $\Phi$

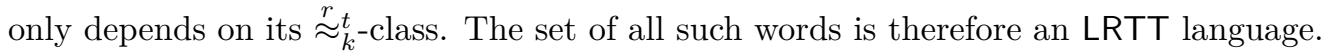

\section{Semigroups with Involution}

In this section we address the question of definability of a language — "is the given reversible regular language definable by a formula in the logic?" — in the previously defined logics. We show that in the case of $\mathrm{FO}$ (bet) the existing theorems provide an algorithm for the problem, while for $\mathrm{FO}(\mathrm{N})$ the answer is not yet known.

First we recall the notion of recognisability by a finite semigroup. A finite semigroup $(S, \cdot)$ is a finite set $S$ with an associative binary operation $\cdot: S \times S \rightarrow S$. If the semigroup operation has an identity, then it is necessarily unique and is denoted by 1 . In this case $S$ is called a monoid. A semigroup morphism from $(S, \cdot)$ to $(T,+)$ is a map $h: S \rightarrow T$ that 
preserves the semigroup operation, i.e. $h(a \cdot b)=h(a)+h(b)$ for $a, b$ in $S$. Further if $S$ and $T$ are monoids the map is a monoid morphism if $h$ maps the identity of $S$ to the identity of $T$.

The set $A^{*}$ (resp. $A^{+}$) under concatenation forms a free monoid (resp. free semigroup). A language $L \subseteq A^{*}$ is recognised by a semigroup (or monoid) $(S, \cdot)$, if there is a morphism $h: A^{*} \rightarrow(S, \cdot)$ and a set $P \subseteq S$, such that $L=h^{-1}(P)$.

Given a language $L$, the syntactic congruence of $L$, denoted as $\sim_{L}$ is the congruence on $A^{*}$,

$$
x \sim_{L} y \quad \text { if } \quad u x v \in L \Leftrightarrow u y v \in L \text { for all } u, v \in A^{*} .
$$

The quotient $A^{*} / \sim_{L}$, (resp. $\left.A^{+} / \sim_{L}\right)$ denoted as $M(L)$, is called the syntactic monoid (resp. syntactic semigroup). It recognises $L$ and is the unique minimal object with this property: any monoid $S$ recognising $L$ has a surjective morphism from a submonoid of $S$ to $M(L)$ [11.

In the particular case of reversible languages the syntactic monoid described above admits further properties. The observation is that the reverse operation can be extended to congruence classes of the syntactic congruence by letting $[x]^{r}=\left[x^{r}\right]$ for each word $x$ and it is well defined since if $x \sim_{L} y$ then $x^{r} \sim_{L} y^{r}$ as can be easily verified. Moreover this operation is an involution, i.e. $\left([x]^{r}\right)^{r}=\left(\left[x^{r}\right]\right)^{r}=\left[\left(x^{r}\right)^{r}\right]=[x]$, and an anti-isomorphism on the congruence classes, i.e. $([x] \cdot[y])^{r}=([x \cdot y])^{r}=\left[(x \cdot y)^{r}\right]=\left[y^{r} \cdot x^{r}\right]=\left[y^{r}\right] \cdot\left[x^{r}\right]=[y]^{r} \cdot[x]^{r}$. Therefore one can enrich the notion of semigroups for recognisability in the case of reversible languages as below.

A semigroup with involution (also called a $\star$-semigroup) $(S, \cdot, \star)$ is a semigroup $(S, \cdot)$ extended with an operation $\star: S \rightarrow S$ (called the involution) such that

1. the operation $\star$ is an involution on $S$, i.e. $\left(a^{\star}\right)^{\star}=a$ for all elements $a$ of $S$,

2. the operation $\star$ is an anti-automorphism on $S$ (isomorphism between $S$ and opposite of $S)$, i.e. $(a \cdot b)^{\star}=b^{\star} \cdot a^{\star}$ for any $a, b$ in $S$.

It is a $\star$-monoid if $S$ is a monoid. It is easy to see that in the case of $\star$-monoids, necessarily $1^{\star}=1$. Clearly the free monoid $A^{*}$ with the reverse operation $r$ as the involution is a $\star$-monoid, since $\left(w^{r}\right)^{r}=w$ and $(v \cdot w)^{r}=w^{r} \cdot v^{r}$. When there is no ambiguity, we just write $A^{*}$ to refer to the $\star$-monoid $\left(A^{*}, \cdot, r\right)$.

A map $h: S \rightarrow T$ between two $\star$-semigroups $(S, \cdot, \star)$ and $(T,+, \dagger)$ is a morphism if it is a morphism between the semigroups $(S, \cdot)$ and $(T,+)$ that preserves the involution, i.e. $h\left(a^{\star}\right)=h(a)^{\dagger}$.

A language $L \subseteq A^{*}$ is said to be recognised by a $\star$-semigroup $(S, \cdot, \star)$, if there is a morphism $h:\left(A^{*}, \cdot, r\right) \rightarrow(S, \cdot, \star)$ and a set $P \subseteq S$, such that $P^{\star}=P$ and $L=h^{-1}(P)$. The following proposition summarises the discussion so far.

- Proposition 12. The following are equivalent for a language $L$.

1. $L$ is a reversible regular language,

2. $L$ is recognised by a finite $\star$-monoid,

3. $M(L)$ with the reverse operation is a finite $\star$-monoid with $P=P^{\star}$ where $P=\{[u] \mid u \in L\}$, i.e. $(M(L), \cdot, r)$ recognises $L$ as a $\star$-monoid.

A semigroup (or monoid) is aperiodic if there is some $n \in \mathbb{N}$ such that $a^{n}=a^{n+1}$ for each element $a$ of the semigroup. Schützenberger-McNaughton-Papert theorem states that a language $L$ is definable in $\mathrm{FO}(<)$ if and only if the syntactic monoid is aperiodic. This theorem in conjunction with Proposition 4 gives that,

- Proposition 13. A reversible language $L$ is definable in $\mathrm{FO}($ bet) if and only if $M(L)$ is aperiodic. 
The above theorem hence yields an algorithm for definability of a language in $\mathrm{FO}$ (bet), i.e. check if the language is reversible, if so compute the syntactic monoid (which is also a monoid with an involution) and test for aperiodicity.

Next we look at the logic $\mathrm{FO}(\mathrm{N})$. The characterisation theorem for $\mathrm{FO}(+1)$ due to Brzozowski and Simon [2], and Beauquier and Pin [1], is stated below. Recall that an element of a semigroup $e$ is an idempotent if $e \cdot e=e$.

- Theorem 14 (Brzozowski-Simon, Beauquier-Pin). The following are equivalent.

1. L is locally threshold testable.

2. $L$ is definable in $\mathrm{FO}(+1)$.

3. The syntactic semigroup of $L$ is finite, aperiodic and satisfies the identity ex $f$ ye $z f=$ ezf ye $x$ for all $e, f, x, y, z \in M(L)$ with $e, f$ idempotents.

Because of Proposition 9 we need to add more identities to characterise the logic $\mathrm{FO}(\mathrm{N})$ in terms of $\star$-semigroups.

- Theorem 15. The syntactic $\star$-semigroup of an $\mathrm{FO}(\mathrm{N})$-definable language satisfies the identity

$$
e x e^{\star}=e x^{\star} e^{\star},
$$

where $e$ is an idempotent, and $x$ is any element of the semigroup.

Proof. Assume we are given an $\mathrm{FO}(\mathrm{N})$-language $L$, with its syntactic $\star$-semigroup $M=$ $\left(A^{+} / \sim_{L}, \cdot, \star\right)$, and $h: A^{+} \rightarrow M$ the canonical morphism recognising $L$. Let $e$ be an idempotent of $M$, and let $x$ be an element of $M$. Pick nonempty words $u$ and $s$ such that $h(u)=e$ and $h(s)=x$.

By definition of the involution, $h\left(u^{r}\right)=e^{\star}$ and $h\left(s^{r}\right)=x^{\star}$. We are going to show that $u s u^{r} \sim_{L} u s^{r} u^{r}$ and hence they will correspond to the same element in the syntactic $\star$-semigroup, proving that $e x e^{\star}=e x^{\star} e^{\star}$.

Since $L$ is $\mathrm{FO}(\mathrm{N})$ definable, we know by Theorem 11 that $L$ is a union of $\stackrel{r}{\approx}_{k}^{t}$ equivalence classes for some $k, t>0$. Consider the words $w=\left(u^{k}\right) s\left(u^{k}\right)^{r}$ and $w^{r}=\left(u^{k}\right) s^{r}\left(u^{k}\right)^{r}$, obtained by pumping the words corresponding to $e$ and $e^{\star}$. Since $e, e^{\star}$ are idempotents, it is clear that $h(w)=h\left(u s u^{r}\right)=e x e^{\star}$ and $h\left(w^{r}\right)=h\left(u s^{r} u^{r}\right)=e x^{\star} e^{\star}$.

For all contexts $\alpha, \beta \in A^{*}$, we show below that $\alpha w \beta \stackrel{r}{\approx}_{k}^{t} \alpha w^{r} \beta$, which implies $\alpha w \beta \in L$ iff $\alpha w^{r} \beta \in L$ since $L$ is a union of $\underset{\approx}{\sim}{ }_{k}^{t}$ classes. It follows that $w \sim_{L} w^{r}$ and therefore $h(w)=h\left(w^{r}\right)$, which will conclude the proof.

Fix some contexts $\alpha, \beta \in A^{*}$. Since $u \neq \varepsilon$, the words $\alpha w \beta$ and $\alpha w^{r} \beta$ have the same prefix of length $k-1$ and the same suffix of length $k-1$. Now, consider $v \in A^{k}$. If an occurrence of $v$ (resp. $v^{r}$ ) in $\alpha w \beta$ overlaps with $\alpha$ or $\beta$ then we have the very same occurrence in $\alpha w^{r} \beta$. Using $w \stackrel{r}{\approx}{ }_{k}^{t} w^{r}$, we deduce that $\sharp^{r}(\alpha w \beta, v)={ }^{t} \sharp^{r}\left(\alpha w^{r} \beta, v\right)$. Therefore, $\alpha w \beta \stackrel{r}{\approx}_{k}^{t} \alpha w^{r} \beta$.

The converse direction is open. The similar direction in the case of $\mathrm{FO}(+1)$ goes via categories 14] and uses the Delay theorem of Straubing [10, 11].

\section{Conclusion}

The logics $\mathrm{MSO}$ (bet), $\mathrm{MSO}(\mathrm{N})$ and $\mathrm{FO}$ (bet) behave analogously to the classical counterparts $\mathrm{MSO}(<), \mathrm{MSO}(+1)$ and $\mathrm{FO}(<)$. But the logic $\mathrm{FO}(\mathrm{N})$ gives rise to a new class of languages, locally-reversible threshold testable languages. The quest for characterising the new class takes us to the formalism of involution semigroups. The full characterisation of the new class is the main question we leave open. Another line of investigation is to study the equationally-defined classes that arise naturally from automata theory. 


\section{References}

1 Danièle Beauquier and Jean-Eric Pin. Languages and scanners. Theoretical Computer Science, 84(1):3-21, 1991.

2 J. A. Brzozowski and Imre Simon. Characterizations of locally testable events. In Proceedings of the 12th Annual Symposium on Switching and Automata Theory (Swat 1971), SWAT '71, pages 166-176, 1971.

3 Siniša Crvenković and Igor Dolinka. Varieties of involution semigroups and involution semirings: a survey. In Proceedings of the International Conference "Contemporary Developments in Mathematics" (Banja Luka, 2000), Bulletin of Society of Mathematicians of Banja Luka, pages 7-47, 2000.

4 Heinz-Dieter Ebbinghaus and Jörg Flum. Finite model theory. Perspectives in Mathematical Logic. Springer, 1995.

5 Andreas Krebs, Kamal Lodaya, Paritosh Pandya, and Howard Straubing. Two-variable logic with a between relation. In Proceedings of the 31st Annual ACM/IEEE Symposium on Logic in Computer Science, LICS '16, pages 106-115, 2016.

6 Andreas Krebs, Kamal Lodaya, Paritosh K. Pandya, and Howard Straubing. An Algebraic Decision Procedure for Two-Variable Logic with a Between Relation. In 27th EACSL Annual Conference on Computer Science Logic (CSL 2018), volume 119 of Leibniz International Proceedings in Informatics (LIPIcs), pages 28:1-28:17, 2018.

7 Andreas Krebs, Kamal Lodaya, Paritosh K Pandya, and Howard Straubing. Two-variable logics with some betweenness relations: Expressiveness, satisfiability and membership. arXiv preprint arXiv:1902.05905, 2019.

8 Robert McNaughton and Seymour A. Papert. Counter-Free Automata (M.I.T. Research Monograph No. 65). The MIT Press, 1971.

9 Marcel Paul Schützenberger. On finite monoids having only trivial subgroups. Information and Control, 8(2):190-194, 1965.

10 Howard Straubing. Finite semigroup varieties of the form $\mathbf{V} \star \mathbf{D}$. Journal of Pure and Applied Algebra, 36:53 - 94, 1985.

11 Howard Straubing. Finite Automata, Formal Logic, and Circuit Complexity. Birkhäuser Verlag, Basel, Switzerland, 1994.

12 Pascal Tesson and Denis Therien. Diamonds are forever: The variety DA. In Semigroups, Algorithms, Automata and Languages, Coimbra (Portugal) 2001, pages 475-500. World Scientific, 2002.

13 Wolfgang Thomas. Classifying regular events in symbolic logic. Journal of Computer and System Sciences, 25(3):360-376, 1982.

14 Bret Tilson. Categories as algebra: An essential ingredient in the theory of monoids. Journal of Pure and Applied Algebra, 48(1-2):83-198, 1987. 